\title{
DIE GRIMSELKRAFTWERKE IN DER BERNEROBERLANDISCHEN LANDSCHAFT
}

\author{
ZU DEN NEUEN ERWEITERUNGSBAUTEN ${ }^{1}$
}

Von Walter Staub

Mit 3 Abbildungen

Das Haslital, das bedeutendste Quertal des Berner Oberlandes, tritt in der Geschichte durch seinen Säumerverkehr über die Grimsel früh hervor. Der Zugang zu diesem stets nur dünn bevölkerten obersten Teile des Aaretals führte zumeist über den Brünig. 1397 wird erstmals ein «Spittel» auf der Grimsel erwähnt. 1734 wurde dieses Hospiz erweitert und ihm eine "Suste» beigefügt. Erst in den neunziger Jahren des vergangenen Jahrhunderts wurde an Stelle des Saumweges die heutige Landstraße. erbaut. Die Haslitaler waren früher reichsfrei als die Bewohner der Urkantone und unterstanden nie auf längere Zeit einem Adligen. So verwalteten sie auch das Grimselhospiz selbst. Dieses lag, bei rund $1900 \mathrm{~m}$ Meereshöhe, wie der $\mathrm{Pa}$, mitten im Aaremassiv. Funde von großen Bergkristallen (1719 am Zinggenstock, 1868 am Tiefengletscher) hatten das Gebiet im 18. und 19. Jahrhundert besonders berühmt gemacht. Die große Zeit aber erlebte der $\mathrm{Paß}$, als um 1840 die Gelehrten L. AgAssiz, E. Desor, C. Vogt, B. Studer auf dem Unteraargletscher über mehrere Sommer hin ersten Studien der Gletscherbewegung oblagen. Die vortrefflich erhaltenen Schlifformen aus der Eiszeit im Aare-Granitgneis machen noch heute die Grimselgegend zum klassischen Gabiet der Glazialforschung.

Kompaktes, undurchlässiges Gestein, unbesiedelte, hochgelegene, ebene Talmulden in felsiger Landschaft, reiche Wasserzuflüsse in einem Quertal mit großem Gefälle ergaben im obern Haslital ideale Vorbedingungen für die Erstellung von WasserAkkumulierbecken zur Produktion von Winterenergie. Die Gelmeralp trug zudem schon vor dem Ausbau einen kleinen Karsee. Vom heutigen Grimselstausee bis Innertkirchen beträgt die Höhendifferenz zirka $1200 \mathrm{~m}$.

Schon 1899 wurde eine Konzession zur Ausnützung der Wasserkräfte im oberen Haslital nachgesucht, jedoch erst 1905, nun durch die Bernischen Kraftwerke, für Verwertung der Kräfte zur Elektrizitätsgewinnung. In zwei Etappen wurden, von den 1925 neugegründeten Kraftwerken Oberhasli AG., erst eine obere Stufe mit der Zentrale Handegg I, dann 1940 eine untere Stufe mit der Zentrale Innertkirchen in Angriff genommen und fertiggestellt.

Die im Oberhasli verfügbaren Wasserkräfte sind sehr bedeutend. Durchschnittlich. fallen bei Guttannen $160 \mathrm{~cm}$, an der Grimsel $200 \mathrm{~cm}$, im Hochgebirge selbst bis $350 \mathrm{~cm}$ Niederschläge im Jahr. Das Einzugsgebiet der Aare umfaßt bis zur Zentrale Handegg $111,5 \mathrm{~km}^{2}$, bis Innertkirchen $342,7 \mathrm{~km}^{2}$. Die jährliche Abflußmenge bis Innertkirchen beträgt $413000000 \mathrm{~m}^{3}$ Wasser. Diese Abflußmengen erleiden im Verlaufe der Jahre relativ nur geringe Schwankungen. Bis zur Handegg ist etwa die Hälfte des Einzugsgebietes mit Firn und Gletscher bedeckt. Diese Gletscher, der Unteraar-, der auf den Unteraarboden mündet, der Oberaar- und der Gauligletscher, wirken abflußregu-

${ }_{1}^{1}$ Der-Direktion der Bernischen Kraftwerke verdanken wir die Klischees sowie die Durchsicht des Manuskriptes, außerdem einen namhaften Beitrag an die farbige Kartenbeilage «Berner Oberland». 
lierend. Ist der Sommer kalt und naß, bleibt in den Höhen ein Teil der Niederschläge als Schnee und Eis liegen; in warmen, trockenen Sommern dagegen ergänzt sich der Abfluß durch die Gletscherschmelze. Zirka 90\% des Jahresabflusses fließen in den fünf Sommermonaten ab. Durch Akkumulierung in Stauseen können diese Abflüsse in großem Ausmaß für die Produktion von Winterenergie nutzbar gemacht werden. Die Oberaaralp und der Unteraarboden münden zudem in engen Talschluchten aus, die durch Talsperren leicht abgeschlossen werden können. In den Kraftwerken des Oberhasli können im Gesamtausbau über 1000 Millionen Kilowattstunden im Jahre erzeugt werden.

Im Sommer 1925 wurde mit den Bauarbeiten an der Grimsel begonnen, und von 1925 bis 1932 war das Tal ein großer Bauplatz mit vorübergehend 1800 Arbeitern.

Die Staumauern waren die Stellen größter Arbeitsverdichtung; auf die Staumauer entfielen im Handeggwerk zirka $60 \%$ der gesamten Baukosten. Für den Transport von über 100000 Tonnen Zement und zirka 80000 Tonnen weiteren Materials zur Fertigstellung dieser ersten Bauetappe mußten neben der Straße spezielle Transporteinrichtungen erstellt werden. Von diesen sind dauernd als Bestandteile der Landschaft eingegliedert: die Bahn Meiringen-Innertkirchen und die Standseilbahn Handegg-Gelmersee. Später, beim Bau des Kraftwerkes Innertkirchen, kam noch die Standseilbahn von Innertkirchen nach dem WasserschloB im Pfaffenkopf dazu; zu erwähnen ist ferner die Freileitung von Guttannen nach Innertkirchen. Die größten Veränderungen im Landschaftsbild aber brachten die Stauseen: eine Riesenkraft der Natur liegt hier gebändigt vor uns, bezwungen durch Menschengeist und Technik.

Der Grimselstausee bleibt auch heute das Kraftzentrum des ganzen Werkes und nimmt den obersten Teil des Haslitales in Anspruch. Er reicht vom Grimselnollen, auf dem heute das neue Hospiz steht, bis zur Zunge des Unteraargletschers. Der See hat eine Spiegelhöhe von $1909 \mathrm{~m}$ über Meer, eine Länge von 5,5 km, eine mittlere Breite von $500 \mathrm{~m}$; die Oberfläche mißt $2,64 \mathrm{~km}^{2}$; das Becken hat einen Stauinhalt von $100 \mathrm{Mil}-$ lionen $\mathrm{m}^{3}$. Der massige Granitriegel des Grimselnollens erleichterte den Talabschluß, der durch den Bau der $114 \mathrm{~m}$ hohen Spitallammauer mit $340000 \mathrm{~m}^{3}$ Mauerkubatur und der Seeufereggsperre erfolgt ist. Diese letztere ist eine geradlinige Schwergewichtsmauer von $42 \mathrm{~m}$ Höhe und $352 \mathrm{~m}$ Länge, über deren Mauerkrone die neue Straße zum heutigen* Hospiz bei $1980 \mathrm{~m}$ Meereshöhe führt. Am Westende liegt der Úberfall des Sees, der in den Herbstmonaten gelegentlich anspricht. Das Wasser wird vom Grimselsee in einem $5220 \mathrm{~m}$ langen Stollen am östlichen Gehänge des Aaretales entlang nach dem Gelmersee geleitet.

Mit Hilfe einer Schwergewichtsmauer von $35 \mathrm{~m}$ Höhe, $384 \mathrm{~m}$ Kronenlänge und $81000 \mathrm{~m}^{3}$ Beton konnte der Spiegel des Gelmersees, eines ehemaligen Karsees, um $30 \mathrm{~m}$ auf $1852 \mathrm{~m}$ Spiegelhöhe gehoben werden, so daß das jetzige Becken einen Nutzinhalt von 13 Millionen $\mathrm{m}^{3}$ besitzt. Durch einen eisengepanzerten Druckschacht gelangt das Wasser nach der Zentrale Handegg I, die bei $1304 \mathrm{~m}$ Meereshöhe in der Talsohle, an einer vor Lawinen und Steinschlag gesicherten Stelle, erbaut wurde. Hier wird es abermals gesammelt und in einem $10 \mathrm{~km}$ langen Stollen dem rechten Talhang entlang nach dem Wasserschloß «Kapf» im Pfaffenkopf oberhalb des Kraftwerkes Innertkirchen geführt. Die Zentrale Innertkirchen ist ganz in den Fels, den Innertkirchner Granit, eingebaut worden; nur die Transformatorenstation steht im Freien. In ihr wird die Energie auf 150000 Volt hinauftransformiert und von da in mächtigen Úberlandleitungen dem Tiefland zugeführt.

Seit der Fertigstellung des ganzen Werkes im Jahre 1943 ist der Mangel an Winterenergie in der Schweiz ständig gewachsen. Die Erzeugung von Winterenergie soll nun 


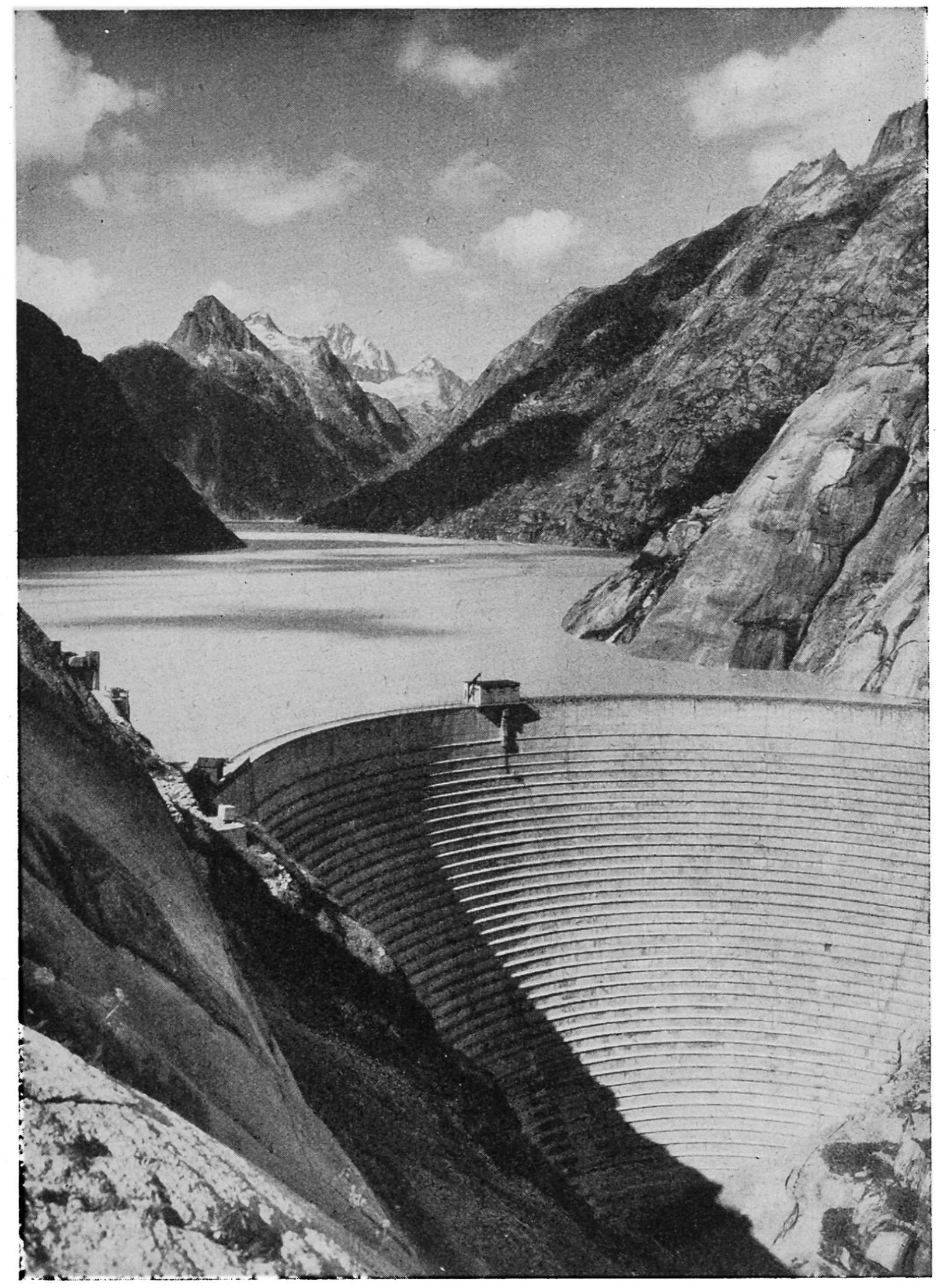

Der Grimselsee mit der $114 \mathrm{~m}$ hohen Spitallammsperre. Im Hintergrund Finsteraarhorn (4274 m); rechts davon, über dem Tierberggletscher mit Escherhorn (3100 m), das Agassizhorn (3953 m); links (schwarz) der Vorder-Zinggenstock $(2920 \mathrm{~m}$ ) 
im Oberhasli verdoppelt werden. Wieder sind landschaftsverändernde, von Seen erfüllte Staubecken in Entstehung. Die Wasser des benachbarten Urbachtales mit dem Gauligletscher werden auf der Mattenalp bei $1850 \mathrm{~m}$ Meereshöhe in einem kleinen Akkumulierbecken gesammelt und durch einen $5800 \mathrm{~m}$ langen Stollen unter dem Ritzlihorn ins Aaretal geleitet. Neben der Zentrale Handegg I mit ihren Wohnhäusern wird eine Zentrale Handegg II erbaut. Der Rätherichsboden, eine wohl glazial ausgeschliffene und zugeschüttete Felsmulde unterhalb der Grimselsperren, wird zum Staubecken umgeformt. Die quer zum Tal verlaufende, im Fels der Gersténegg verankerte Sperre bei $1767 \mathrm{~m}$ Meereshöhe wird $84 \mathrm{~m}$ hoch und an der Krone $34 \mathrm{~m}$ lang sein. Das Seebecken wird 27 Millionen $\mathrm{m}^{3}$ Wasser fassen können. Ergeben Handegg I und

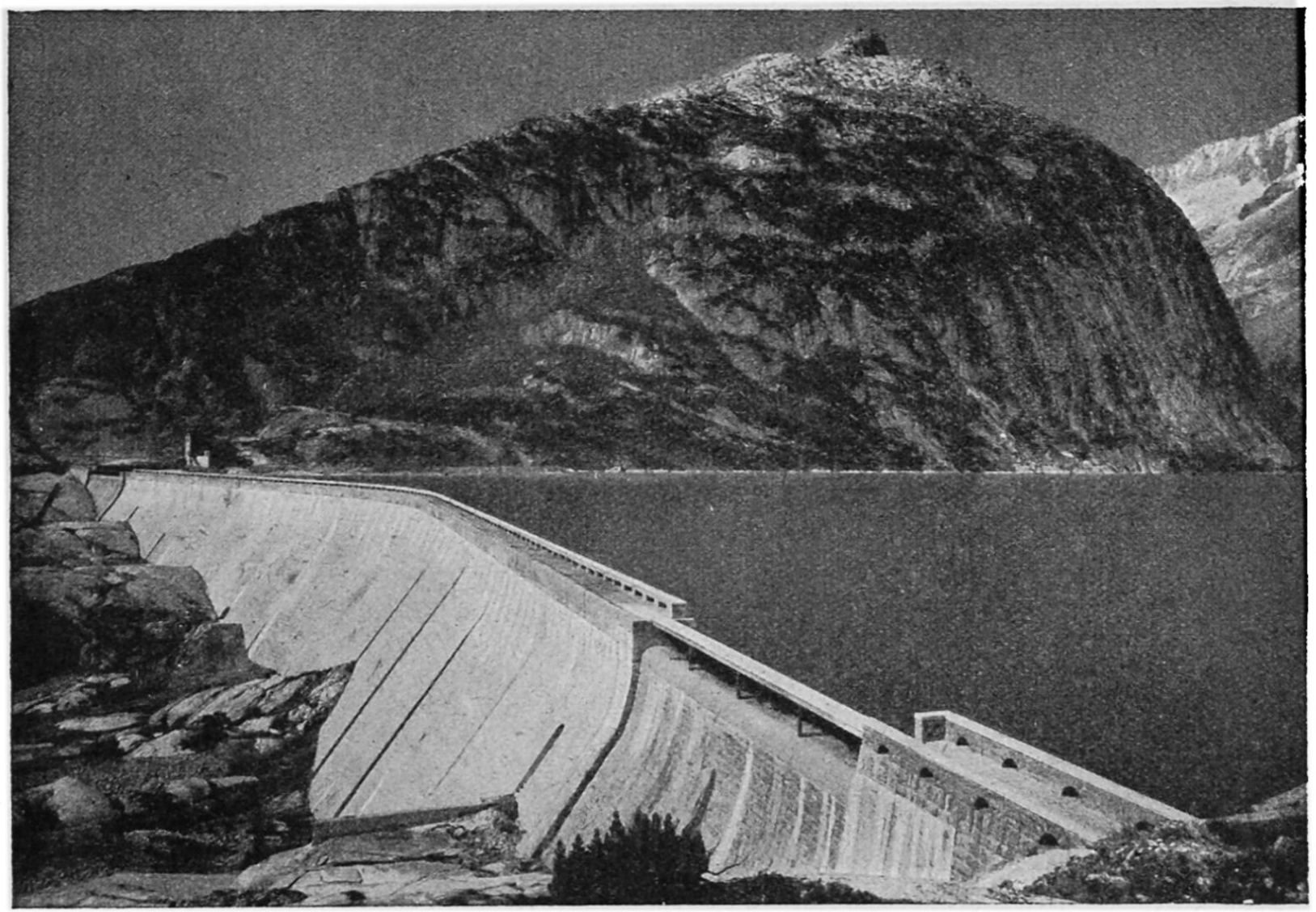

Der Gelmersee mit der $35 \mathrm{~m}$ hohen Staumauer

die Zentrale Innertkirchen 600 Millionen $\mathrm{kWh}$ gleichmäßiger Jahresenergie plus 70 bis 100 Millionen kWh zusätzlicher Sommerenergie, so können nun die Neuanlagen noch dazu 100 Millionen kWh Winterenergie und zirka 140 Millionen kWh Sommerenergie crzeugen. Die Energie der beiden Handeggzentralen soll in Zukunft daselbst direkt auf 150000 Volt transformiert und teils mittels einer Freileitung, teils mittels Kabel über Guttannen nach Innertkirchen gebracht werden.

Die Schaffung des Stausees Rätherichsboden macht bei der Sperrstelle die Verlegung der Grimselstraße notwendig, die erst beim Einfluß der Aare in den neuen See wieder in die alte Straße einmündet. Der alte, historische Grimselsaumweg bleibt erhalten. Er führt aber künftig statt über den Rätherichsboden in diesem Abschnitt am linken Talhang dem neuen See entlang. 


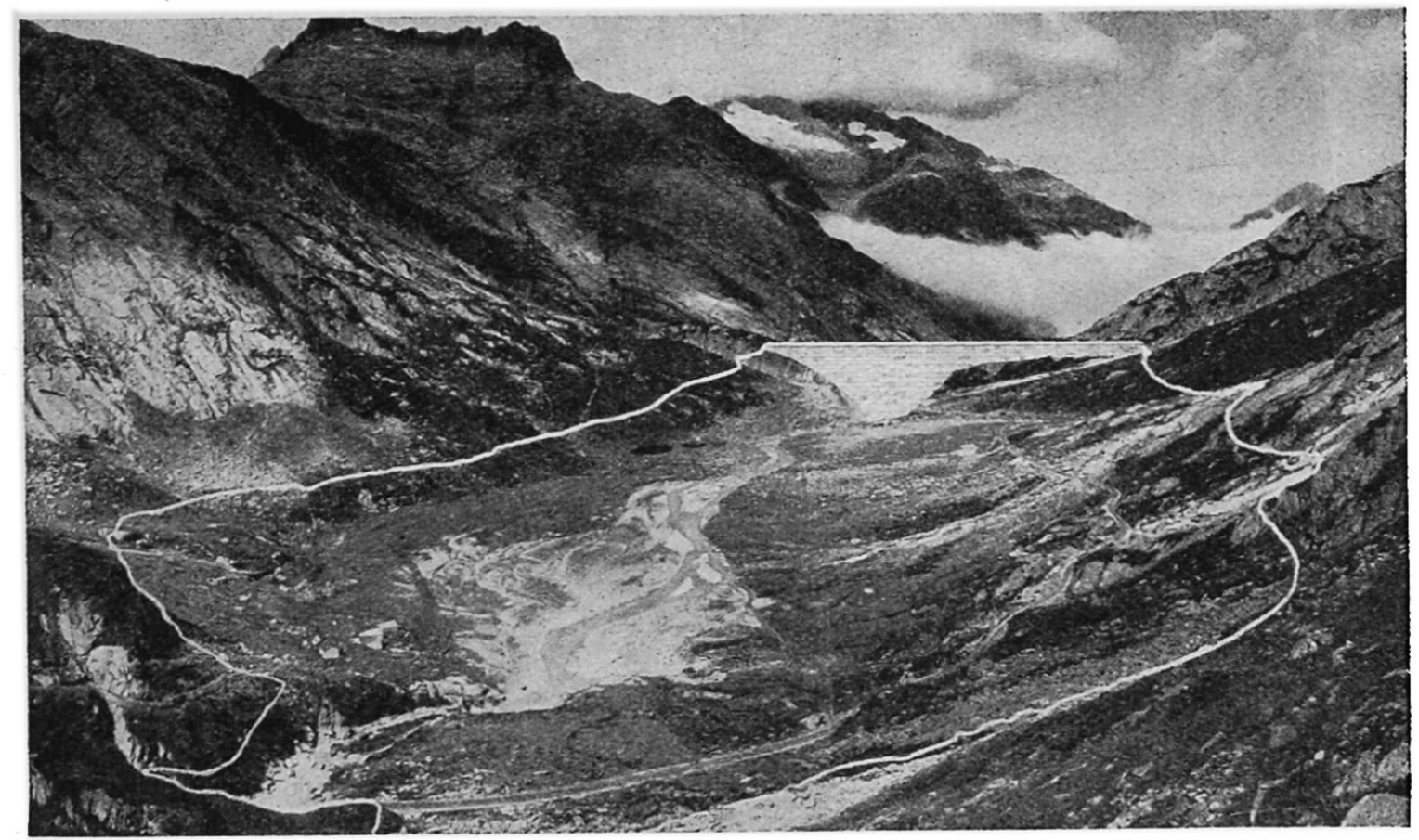

Der Rätherichsboden unterhalb der Grimselsperren. Blick talabwärts. Die weiße Linie gibt die Grenze des künftigen Stausees an

Die Inbetriebnahme des neuen Kraftwerkes Handegg II ist auf den Sommer 1950 vorgesehen. Für das Jahr 1951/52 ist die Erstellung eines weiteren Stausees auf der Oberaaralp geplant, der die Produktion von Winterenergie um weitere 220 Millionen $\mathrm{kWh}$ erhöhen soll, ebenso eine Stauung des Totensees auf der Paßhöhe.

Mit diesen Bauten wird das Haslital technisch weitgehend umgeformt sein und den Charakter einer Kraftwerklandschaft erhalten, in vermehrtem Maße anziehend für Ingenieure, doch auch für Bergfreunde.

Literatur: Grimsel, Kraftwerkbauten im Oberhasli. Innertkirchen 1948.

\section{LES FORCES MOTRICES DE L'OBERHASLI COMME PARTIE CONSTITUANTE DU PAYSAGE DE L'OBERLAND BERNOIS}

La partie supérieure de la vallée de l'Aar, le Hasli, semble être prédestinée à la construction d'usines électriques. Après avoir terminée l'usine de la Handegg avec le grand lac de la Grimsel et celui de Gelmer (en 1925-1932), l'usine d'Innertkirchen (bâtie entièrement dans le rocher, en 1940-1943), la demande de force électrique d'hiver a augmenté constamment. C'est pour cette raison, la compagnie a décidé un nouvel agrandissement de l'entreprise. La plaine de Rätherichsboden sera inondée; l'eau de la vallée d'Urbach sera conduite par un tunnel au-dessous de la chaine du Ritzlihorn jusqu'à la Handegg. Ainsi plus de 1000 millions $\mathrm{k}$ Wh pourront être produites annuellement dans toute la vallée supérieure de l'Aar.

\section{GLI IMPIANTI IDROELETTRICI NEL PAESAGGIO DELL'OBERLAND BERNESE}

Il corso superiore dell'Aare, l'Haslital, sembra predestinato alla costruzione di impianti idroelettrici. Dalla costruzione dell'impianto idroelettrico di Handegg con i bacini dei laghi del Grimsel e di Gelmer (1925-1932) e di quello di Innertkirchen (1940-1943), il consumo di energia elettrica invernale fu in continuo aumento, di modo che i Berner Kraftwerke decisero un ampliamento dello sfruttamento. Il piano del Rätherichsboden verrà occupato da un bacino e l'acqua della Valle di Urbach sarà convogliata per mezzo di una galleria attraverso la catena del Ritzlihorn fino ad Handegg. L'Aare superiore potrà così dare complessivamente più di 1000 milioni di $\mathrm{kWh}$ all'anno. 